\title{
The N-terminal domain of Escherichia coli RecA have multiple functions in promoting homologous recombination Chien-Der Lee and Ting-Fang Wang*
}

\author{
Address: Institute of Molecular Biology, Academia Sinica, Taipei 115, Taiwan \\ Email: Chien-Der Lee - cdlee@gate.sincia.edu.tw; Ting-Fang Wang* - tfwang@gate.sinica.edu.tw \\ * Corresponding author
}

Published: I April 2009

Journal of Biomedical Science 2009, 16:37 doi:10.1/86/1423-0127-16-37

This article is available from: http://www.jbiomedsci.com/content//6/I/37

(c) 2009 Lee and Wang; licensee BioMed Central Ltd.

This is an Open Access article distributed under the terms of the Creative Commons Attribution License (http://creativecommons.org/licenses/by/2.0), which permits unrestricted use, distribution, and reproduction in any medium, provided the original work is properly cited.
Received: 18 February 2009

Accepted: I April 2009

\begin{abstract}
Escherichia coli RecA mediates homologous recombination, a process essential to maintaining genome integrity. In the presence of ATP, RecA proteins bind a single-stranded DNA (ssDNA) to form a RecA-ssDNA presynaptic nucleoprotein filament that captures donor double-stranded DNA (dsDNA), searches for homology, and then catalyzes the strand exchange between ssDNA and dsDNA to produce a new heteroduplex DNA. Based upon a recently reported crystal structure of the RecA-ssDNA nucleoprotein filament, we carried out structural and functional studies of the N-terminal domain (NTD) of the RecA protein. The RecA NTD was thought to be required for monomer-monomer interaction. Here we report that it has two other distinct roles in promoting homologous recombination. It first facilitates the formation of a RecA-ssDNA presynaptic nucleoprotein filament by converting ATP to an ADP-Pi intermediate. Then, once the RecA-ssDNA presynaptic nucleoprotein filament is stably assembled in the presence of ATP $\gamma$, the NTD is required to capture donor dsDNA. Our results also suggest that the second function of NTD may be similar to that of Arg243 and Lys245, which were implicated earlier as binding sites of donor dsDNA. A two-step model is proposed to explain how a RecA-ssDNA presynaptic nucleoprotein filament interacts with donor dsDNA.
\end{abstract}

\section{Background}

Escherichia coli RecA is the founding member of the RecA protein family. It is essential for the initiation of repair of DNA breaks via homologous recombination, induction of the DNA damage-induced 'SOS' response, and activation of translesion DNA synthesis, as well as development and transmission of antibiotic resistance genes $[1,2]$. Nearly all known functions of RecA require the formation of a presynaptic helical filament comprised of single-stranded DNA (ssDNA) bound to multiple RecA monomers with ATP. During homologous recombination, this activated form of the helical filament is capable of interacting with homologous double-stranded DNA (dsDNA) to form a heteroduplex DNA molecule. Eventually, the DNA strands are exchanged, resulting in the displacement of one of the original duplex strands and the subsequent creation of a new heteroduplex (or D-loop). This function is evolutionarily conserved in other members of the RecA family, including archaeal RadA and the eukaryotic proteins, Rad51 and Dmc1.

The RecA monomer has three major structural domains: a small N-terminal domain (NTD), a core ATPase domain (CAD), and a large C-terminal domain (CTD). By contrast, the monomers of RadA/Rad51/Dmc1 consist of a CAD and a larger NTD. The CAD, often referred to as the 
RecA fold [3], is structurally similar to the ATPase domains of DNA/RNA helicases, F1 ATPases, chaperonelike ATPases, and membrane transporters [4]. Highly conserved in all RecA family proteins, the CAD contains two disordered loops (denoted the L1 and L2 motifs) that bind to ssDNA and are responsible for ssDNA-stimulated ATPase activity [5]. All RecA family proteins are polymerized via a polymerization motif located between the NTD and the CAD. The polymerization motif contains a hydrophobic residue (isoleucine 26 in E. coli RecA; phenylalanines in RadA, Rad51, and Dmc1) that docks within the hydrophobic pocket of the neighboring CAD. This interaction was also observed at the binding interface between a human Rad51 monomer and a BRC repeat of BRCA2 tumor suppressor protein [6-9]. The polymerization motif of archaeal RadA protein is responsible for the assembly of different quaternary structures, including toroidal rings, as well as right-handed and left-handed helical filaments $[10,11]$.

The crystal structures of RecA-ssDNA and RecA-dsDNA nucleoprotein complexes with $\mathrm{ADP}-\mathrm{AlF}_{4}-\mathrm{Mg}^{2+}$ have recently been reported [12]. These new structures have provided unprecedented new insights into the mechanisms and energetics of RecA protein. In the RecA-ssDNA$\mathrm{ADP}-\mathrm{AlF}_{4}-\mathrm{Mg}^{2+}$ filament complex, the ssDNA is bound by the L1 and L2 loop regions as well as by the N-terminal portion of the $\alpha \mathrm{F}$ and $\alpha \mathrm{G}$ helices that follow L1 and L2, respectively. The ADP- $\mathrm{AlF}_{4}-\mathrm{Mg}^{2+}$ is sandwiched between the CADs of two adjacent RecA protomers in a completely buried environment. $\mathrm{AlF}_{4}$ - group is coordinated by the side chains of Lys248 and Lys250. Lys250 also hydrogen bonds to the side chain of Glu96 in the neighboring RecA protomer. Glu96 is the catalytic residue thought to activate a water molecule for nucleophilic attack on the $\gamma$ phosphate. This second interface is absent in the inactive filament, where the corresponding interface of the ATP analogue, adenylyl-imidodiphosphate (AMP-PNP), is solvent exposed. Therefore, the charged-stabilized hydrogen bonds that Lys 248 and Lys250 make to the $\mathrm{AlF}_{4}$ - group could explain the ATP-dependency of DNA binding, because the $\gamma$-phosphate of ATP is sensed across the RecARecA interface cooperating with DNA binding to promote the transition to the active filament state. Close to the filament axis, the ssDNA is extended $50 \%$ in length relative to B-form DNA with the same sequence. Each RecA monomer interacts with three nucleotides of the DNA (a triplet), and each triplet is also bound by three contiguous RecA monomers. Strikingly, the DNA-RecA interaction, or DNA extension, is not isotropic at the nucleotide level; instead, the DNA comprises a three nucleotide segment with a nearly normal B-form distance between bases (an axial rise of 3.5-4.2 $\AA$ for ssDNA and 3.2-4.5 $\AA$ for dsDNA), followed by a long, untwisted internucleotide stretch ( 7.1-7.8 $\AA$ in ssDNA and $8.4 \AA$ in ssDNA) before the next nucleotide triplet, in a repeating pattern. Such an unusual repeat pattern of DNA extension reveals a new structural basis of the dynamics of filament assembly in the presence of ssDNA, including initiation (or nucleation) of filament assembly and the observed cooperativity of RecA-DNA binding.

The RecA-dsDNA-ADP-AlF ${ }_{4}^{-}-\mathrm{Mg}^{2+}$ crystal structure was postulated to be an end product after the strand exchange reaction between a RecA-ssDNA nucleoprotein filament and a homologous dsDNA target $[12,13]$, implying that RecA protein filaments may complete all functions (including ssDNA binding, donor dsDNA capturing, and strand exchange) in right-handed forms and also within the filament axes. Here, we considered an alternative possibility: that the RecA-dsDNA crystal structures might simply represent annealing products of the ssDNA in the RecA-ssDNA nucleoprotein filament and a complementary ssDNA. First, in the RecA-ssDNA filament structure, the purine and pyrimidine bases of bound ssDNA are outwardly exposed. Second, the complementary ssDNA in the RecA-dsDNA structure makes very few physical contacts with the RecA protein filament, indicating that the annealing of these two ssDNAs has very little impact on the protein structures. Indeed, the overall protein structures of RecA-dsDNA filaments are highly similar to those of RecA-ssDNA-ADP-AlF ${ }_{4}^{-} \mathrm{Mg}^{2+}$ structures $[12,13]$. Because the molecular mechanism of the strand exchange reaction is still not understood, it is important to examine these two different possibilities further.

The RecA-ssDNA crystal structures also indicate that Arg243 and Lys245 might constitute a binding site for the donor dsDNA during the strand exchange reaction. Arg243 and Lys 245 are $25 \AA$ away from the filament axis and have a repeat distance of $\sim 28 \AA$ along the filament axis. Their positively charged side chains are solventexposed and face towards the central axis of the RecAssDNA helical filament (Figure 1A) [12]. This is consistent with previous reports that these positively charged residues are responsible for dsDNA capture during homology pairing and strand exchange $[14,15]$. However, a closer look at the RecA-ssDNA crystal structure revealed that the positively charged side chains of Arg243 and Lys245 are not exposed to the exterior surface of the nucleoprotein filament; they reside inside the filament (Figure 1B). We speculated that these two amino acid residues might not be solely responsible for dsDNA recruitment. Other structural element(s), located at the outermost surface of the RecA-ssDNA presynaptic filament, may assist the RecAssDNA presynaptic filament in its search for donor dsDNA.

In the present study, we report that the NTD of RecA may have such a function. Additional mutant analysis revealed 

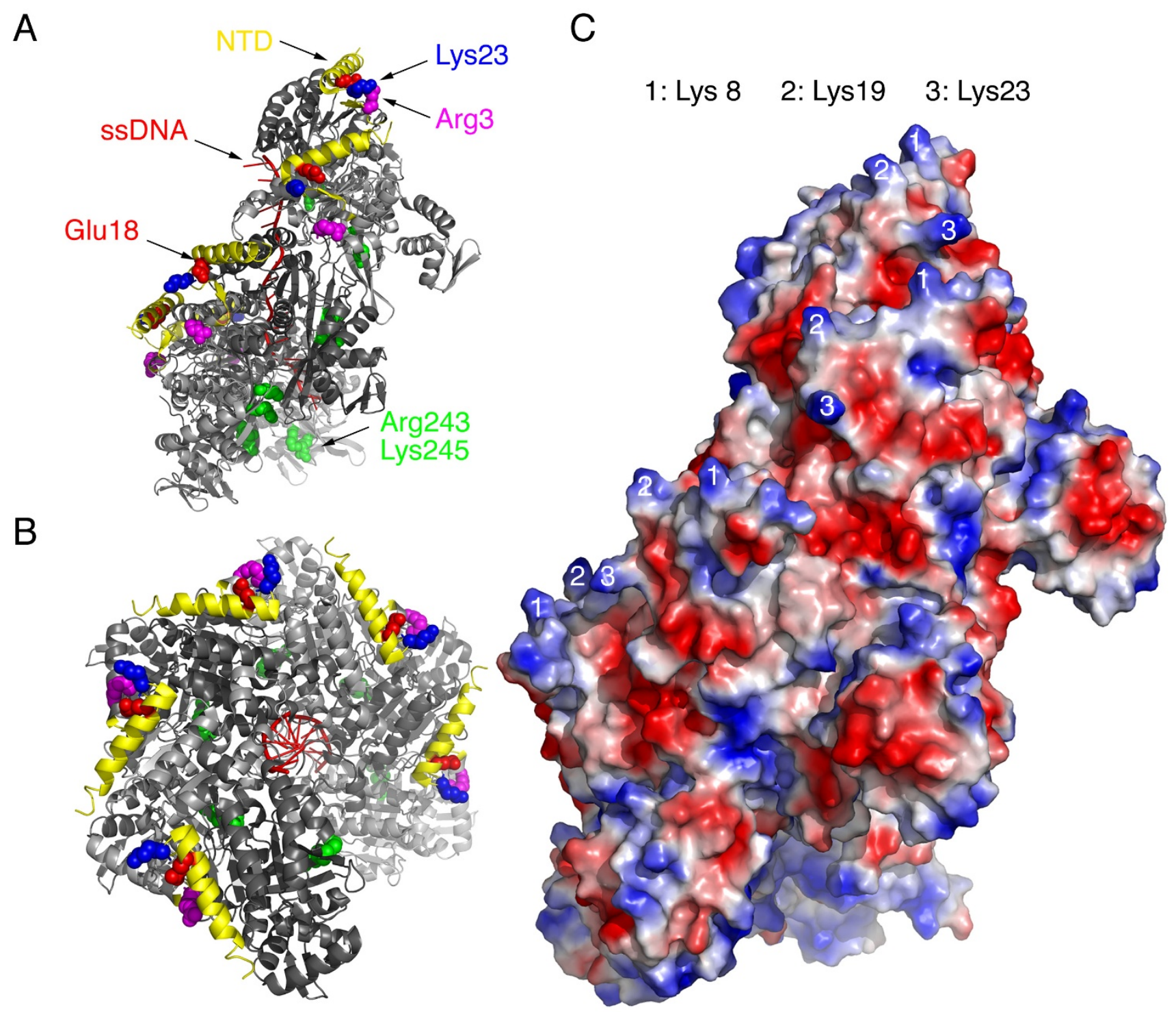

Figure I

Structure of a RecA-ssDNA-ADP-AIF $4-\mathbf{M g}^{2+}$ presynaptic nucleoprotein filament. Shown are ribbon diagrams of side $(A)$ and top $(B)$ views of the recently reported 3CMU crystal structure [12]. The NTDs and ssDNA are shown in yellow and red, respectively. The side chains of three charged residues are depicted as a ball-and-stick model. (C) Surface charge potential of the 3CMU RecA-ssDNA-ADP-AIF $4^{-}-\mathrm{Mg}^{2+}$ presynaptic nucleoprotein filament. The positively and negatively charged regions are indicated in blue and red, respectively. The positions of Lys8 [I], Lys 19 [2], and Lys23 [3] are indicated.

that it might have at least two distinct roles in promoting the RecA-mediated homologous recombination reaction.

\section{Materials and methods}

RecA protein production, enzymatic assays, and DNA substrates

An improved SUMO fusion protein expression system [16] was used to rapidly produce RecA proteins in E. coli. The nuclease assay, D-loop formation assay, ssDNAdependent ATPase activity assay, and DNA substrates used in this study have also been described in detail [16]. ATP $\gamma \mathrm{S}$ (Adenosine 5'-O-(3-thio)triphosphate) and AMPPNP were purchase from Sigma Aldrich.

\section{Electron microscopy}

The wild-type and mutant E. coli RecA proteins $(2 \mu \mathrm{M})$ were incubated with $4 \mu \mathrm{M}$ circular $\Phi X 174$ dsDNA (in base pairs [bps]) at $37^{\circ} \mathrm{C}$ for $30 \mathrm{~min}$ in reaction buffer $(1 \mathrm{mM}$ ATP $\gamma \mathrm{S}, 10 \mathrm{mM} \mathrm{Mg}{ }^{2+}$-acetate, $100 \mathrm{mM} \mathrm{Na}$ acetate, $25 \mathrm{mM}$ Tris-Cl pH 7.4), and were chilled on ice to stop the reac- 
tion. A droplet $(4 \mu \mathrm{l})$ was placed on a copper grid (300 mesh, Pelco, USA) coated with fresh carbon for $1 \mathrm{~min}$ at room temperature. Excess buffer was then carefully blotted away from the edge of the grid with Whatman \# 1 filter paper (Whatman Inc., USA). After staining for 4 min with 2.5\% uranyl acetate, excess liquid was removed and the samples were air dried at room temperature. Bio-transmission EM was performed with a Tecnai G2 Spirit Bio TWIN (FEI Co., Netherlands) using an acceleration voltage of $120 \mathrm{kV}$. Images were recorded with a slowscan CCD camera (Gatan MultiScan 600) at a resolution of at least 1024 $\times 1024$ pixels.

\section{Duplex DNA capture assay}

The duplex DNA capture assay was carried out using a protocol modified from that described previously for Rad51 and Hop2-Mnd1 proteins [17]. To obtain presynaptic filaments for the dsDNA capture assay, a 5'-biotinylated ssDNA PA1656 (15 $\mu \mathrm{M}$ in nucleotides) and RecA proteins (5 or $20 \mu \mathrm{M}$ ) were mixed with $4 \mu \mathrm{L}$ streptavidin-coated magnetic beads (Novagen, USA) in $20 \mu \mathrm{L}$ of buffer C (20 $\mathrm{mM}$ HEPES-KOH at pH 7.0, $1 \mathrm{mM}$ DTT, $100 \mathrm{ng} / \mathrm{mL}$ BSA, $2 \mathrm{mM} \mathrm{Mg}^{2+}$-acetate, 5\% glycerol and $1 \mathrm{mM} \mathrm{ATP} \gamma \mathrm{S}$ ) for 5 $\min$ at $37^{\circ} \mathrm{C}$. BSA was used as a negative control for RecADNA binding reactions. The magnetic beads were isolated with a magnetic separator (Novagen). The supernatant (denoted as "S1") was set aside for later analysis. The magnetic beads were washed twice with $20 \mathrm{~mL}$ buffer $\mathrm{C}$, and then mixed with $15 \mu \mathrm{M}$ of donor dsDNA (bps) in $20 \mu \mathrm{L}$ of buffer D (20 mM HEPES-KOH at pH 7.0, $1 \mathrm{mM} \mathrm{DTT,} 11$ $\mathrm{mM} \mathrm{Mg}{ }^{2+}$-acetate, $5 \%$ glycerol) at $37^{\circ} \mathrm{C}$ for $10 \mathrm{~min}$ with gentle mixing every $1 \mathrm{~min}$. This donor dsDNA was 300 bps in length, and its central region was homologous to PA1656. The magnetic beads were isolated again with a magnetic separator. The supernatant (denoted as "S2") was set aside for later analysis. After the magnetic beads were washed twice with $20 \mu \mathrm{L}$ of buffer E $(20 \mathrm{mM}$ HEPES$\mathrm{KOH}$ at pH 7.0, $1 \mathrm{mM}$ DTT, $11 \mathrm{mM} \mathrm{Mg}^{2+}$-acetate, 5\% glycerol and $1 \mathrm{mM} \mathrm{ATP} \gamma \mathrm{S}$ ), proteins and DNA substrates were eluted by incubating with $20 \mathrm{~mL}$ of $1 \%$ SDS. The SDS eluates (denoted as "B") were separated on either a 12\% SDSPAGE stained with Coomassie blue to visualize bovine serum albumin (BSA) and RecA proteins, or a 1.5\% agarose gel stained with ethidium bromide to visualize the donor dsDNA.

\section{Results}

\section{The NTD of RecA is similar in amino acid sequence to $a$ functional motif of RadA/Rad5 I/Dmc I}

The NTD of E. coli RecA contains only 33 amino acid residues and is much smaller in size than the RadA/Rad51/ Dmc1 NTDs (> 60 amino acids). The crystal structure of the RecA-ssDNA presynaptic filament reveals that NTDs are located at the exterior surface of the helical filament. Moreover, NTDs are not directly involved in RecA-ssDNA interaction or RecA-ATP binding (Figure 1A) [12]. A superimposition of RecA-RecA pairs from the active RecAssDNA presynaptic filament onto an inactive RecA protein filament (achieved by aligning the CADs) revealed a large conformational change in the hinge region (residues 31$40)$ that connects the NTD and CAD. As a result, these two filaments differ by a $32^{\circ}$ rotation and an $18.5 \AA$ translation of the CAD [12]. This hinge region is located immediately after the polymerization motif (i.e., Ile26 of E. coli RecA), which functions as a fulcrum to mediate a large conformational change in response to ssDNA binding [12]. A similar scenario was also reported in a structural transition of the RecA protein filament from a compressed form to a relaxed form [10]. Intriguingly, RadA/Rad51/ Dmc1 protein also contains a similar structural motif that we referred to previously as the subunit rotation motif (SRM) [18]. The SRM also uses the polymerization motif as a fulcrum to mediate rotation along the central axis of the archaeal RadA protein polymer. Continuous clockwise axial rotation of archaeal RadA proteins is responsible for the progression of stepwise structural transitions: first from an inactive ring to a right-handed filament with 6 monomers per helical pitch, then to an overextended right-handed filament with 3 monomers per helical pitch, and, finally, to a left-handed filament with 4 monomers per helical pitch $[10,11,18]$. A key consequence of these structural transitions is the progressive relocation of the NTDs and the L1 ssDNA binding motif. The NTD has been shown to mediate donor dsDNA binding in both human Rad51 [19] and archaeal RadA [18]. In the RadA right-handed helical filament with 6 monomers per helical pitch, L1 resides near the axis filament and the NTD is located at the exterior surface of the filament. In contrast, in the overextended right-handed helical filament with 3 monomers per helical pitch, L1 relocates to the exterior surface of the filament and constitutes an outward-opening palm structure in combination with the NTD. Inside this palm structure, 5 conserved basic amino acid residues (Lys27 and Lys60 of the NTD; and Arg117, Arg223, and Arg229 of the L1 motif) of Sulfolobus solfataricus RadA (SsoRadA) surround this pocket ( $25 \AA$ in diameter), which may be wide enough to simultaneously accommodate an ssDNA and a donor dsDNA [18]. All five positively charged residues are evolutionarily conserved in all archaeal and eukaryotic RecA family proteins. Therefore, the overextended right-handed filament structure of SsoRadA was proposed to represent a structural intermediate during the homologous search and pairing process of archaeal and eukaryotic RecA family proteins [18]. Similarly, as described above, the RecA-ssDNA-ADP-AlF 4 -$\mathrm{Mg}^{2+}$ nucleoprotein filament was also overextended $[12,13]$.

The NTD of RecA was previously considered to be structurally and functionally distinct from those of Rad51/ 
Dmc1/RadA proteins. The RecA NTD contains only a helix (residues 1-23) and a $\beta$-loop (residues 24-33). By contrast, the NTDs of archaeal and eukaryotic RecA family proteins are composed of two helix-hairpin-helix ( $\mathrm{HhH})$ motifs, in which a pseudo two-fold unit is composed of two HhH motifs linked by a connector $\alpha$-helix. The $\mathrm{HhH}$ motifs and the connector $\alpha$-helix are denoted as H1' $h$ 'H2', $\mathrm{H} 1 \mathrm{hH} 2$, and Hc. Each HhH motif contains two helices (denoted as H1, H1', H2, or H2') and a hairpin (denoted as $h$ or $\left.h^{\prime}\right)$ [18]. We reported earlier that the second H1hH2 motif of SsoRadA (i.e., the $\alpha 3-\beta 3-\alpha 4$ region indicated in Figure 2A) is located at the outer surface of the NTD and constitutes a positively charged patch that is responsible for dsDNA binding. Intriguingly, we found that the $\alpha$-helix of the RecA NTD (residues 1-23) shows significant amino acid sequence conservation with the second H1hH2 motif of archaeal and eukaryotic RecA family proteins. First, Gly15 and Lys 23 of RecA are con-

A

Rad51/Dmc1/RadA NTD

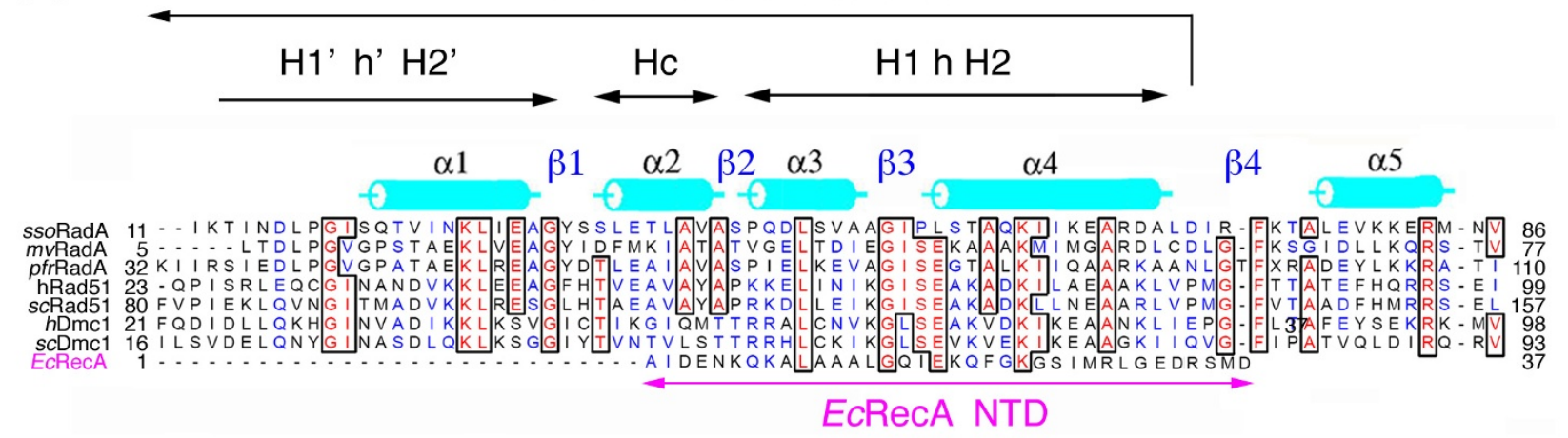

B
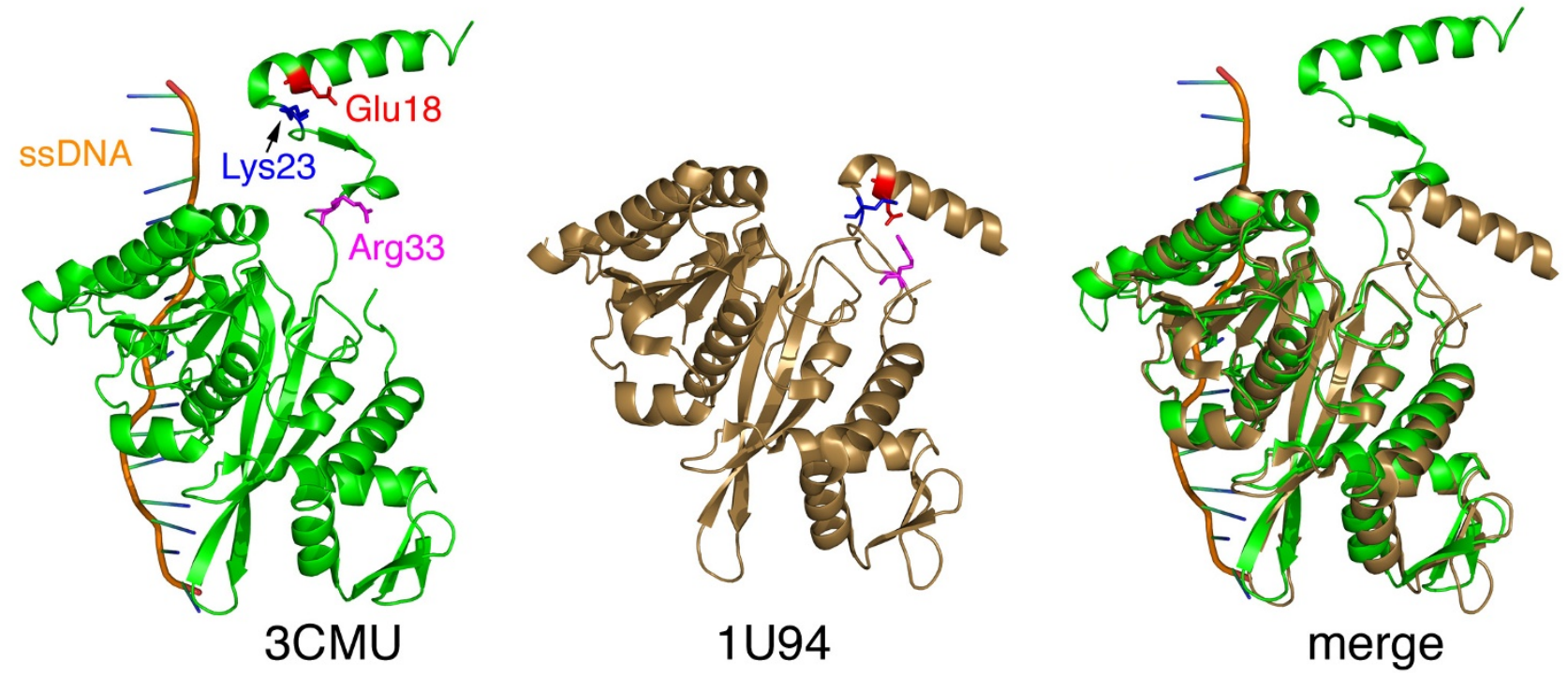

Figure 2

The E. coli RecA NTD exhibits significant amino acid sequence homology with the NTDs of the homologous proteins Rad5 I/Dmc I/RadA. (A) Sequence alignment of the NTDs of RecA proteins from S. solfataricus (SsoRadA), M. voltae (MvRadA), P. furiosus (PfRad5I), H. sapiens (HsRad5I and HsDmcl), S. cerevisiae (ScDmcl and ScRad5I), and E. coli (EcRecA). Secondary structural features of the left-handed SsoRadA helical filament are indicated in cyan $(\alpha-$ helices $)[10]$. Functional motifs are indicated under their corresponding amino acid sequences: the first $\mathrm{HhH}$ motif $\left(\mathrm{H}^{\prime} \mathrm{h}\right.$ ' $\left.\mathrm{H} 2 \mathrm{\prime}\right)$, core helix (Hc the), and the second $\mathrm{HhH}$ motif $(\mathrm{HIHh} 2)$. (B) The NTD carries out a large conformational change in response to ssDNA and ATP $\gamma$ S. Shown are ribbon diagrams of the monomeric RecA structures in the RecA-ssDNA-ADP-AIF ${ }_{4}^{-}$Presynaptic filament [I 2] and in the inactive RecA protein helical filament [3]. The ssDNA and RecA polypeptides are shown in gold and green, respectively. The side chains of Glu 18, Lys23, and Arg33 are depicted as ball-and-stick models. 
served in all RecA family members listed in Figure 2A. The equivalent respective residues in SsoRadA are Gly52 and Lys60, and in human Rad51 are Gly65 and Lys73. Importantly, Gly65 of human Rad51 and Lys60 of SsoRadA were both implicated previously in dsDNA binding $[18,19]$. Second, Lys 8 of E. coli RecA also conserved in eukaryotic Rad51 and Dmc1 proteins. The positively charged side chains of Lys8, Lys19, and Lys23 all point outward to the outermost surface of the active RecA-ssDNA presynaptic nucleoprotein filament (Figure 1C) [12]. Intriguingly, these three lysine residues are conserved among most, if not all, prokaryotic RecA proteins [20]. Third, Glu18 of RecA is also conserved in several other RecA family proteins (Figure 2A). Glu18 may have a role in RecA interaction with ATP or ssDNA. It forms a salt bridge with Arg33 in a compressed/inactive form of the RecA protein filament [3], (i.e., the 1U94 structure, shown in the middle panel of Figure 2B). This salt bridge falls apart in the active/extended RecA-ssDNA-ADP-AlF ${ }_{4}^{-}-\mathrm{Mg}^{2+}$ presynaptic filaments (3CMU; Figure 2B, right panel) [12]. In the latter case, Glu18 makes hydrogen bonds with Ser25, and Arg33 forms a salt bridge with Glu35. The electrostatic charges of Glu18, Arg33, and Glu36 are diminished or neutralized. As a result, only the positive side chains of Lys8, Lys19, and Lys23 are exposed on the outer surface of the RecA NTD (Figure 1C).

\section{Production of the wild type and mutant RecA proteins}

To functionally characterize the NTD of RecA, an improved SUMO fusion protein expression system that we developed recently [16] was applied to produce a panel of RecA mutant proteins. Each mutant protein carries one or two point mutations in Glu18, Lys23, or Arg33. To our knowledge, these NTD mutant proteins have not been properly examined before [21]. Native wild-type RecA protein has 352 amino acids, beginning with an alanine. Edman degradation confirmed that the $\mathrm{N}$-terminus of purified wild-type RecA protein was identical to the expected amino acid sequence [16]. Although the purified RecA protein looked reasonably pure (Figure $3 \mathrm{~A}$ ), we still used a 5'-end ${ }^{32}$ P-labeled ssDNA substrate (PA1656, 50 nucleotides) to determine whether it was contaminated with nuclease. ExoI was used as a positive control for the nuclease assay, and all 5'-end ${ }^{32}$ P-labeled ssDNA substrates were degraded after incubation with ExoI for $30 \mathrm{~min}$. In contrast, no purified RecA proteins cleaved any 5'-end ${ }^{32} \mathrm{P}$-labeled ssDNA substrate under the same conditions (Figure 3B). Therefore, nuclease contamination was not a problem in our production protocol. We confirmed that the purified wild-type RecA was catalytically active in various biochemical assays, and that its activity was indistinguishable from commercially obtained RecA [16]. We also showed by electron microscopy that all NTD mutant proteins examined in this analysis could form helical filaments or protein rings (Figure
3C), indicating they had no apparent defect in polymerization or in formation of the nucleoprotein filament.

\section{Functional characterization of NTD mutant proteins}

D-loop formation assays were performed with a 5 '-end 32P-labeled ssDNA PA1656 and a supercoiled dsDNA GW1 as previously described [16]. When ATP or AMPPNP (a non-hydrolyzable ATP analogue) was used as a nucleotide cofactor, four mutants (K23A, K23E, R33A, and R33E) produced no or very little D-loop product. By contrast, E18A and E18K single mutants, and the E18K/ K23A double mutant produced, respectively, 20\%, $\sim 187 \%$, and $\sim 90 \%$ of the amount of D-loop products made by wild-type RecA (Figure 4). The gain-of-function phenotype of E18K was strong enough to rescue the K24A mutation under the same conditions. These results suggest that positively charged side chains at the outer surface of the NTD are essential for RecA's function.

Surprisingly, when the same assay was performed in the presence of ATP $\gamma \mathrm{S}$, all the RecA-NTD mutant proteins that were examined became catalytically active (Figure $4 \mathrm{C}$ ). Although ATP $\gamma \mathrm{S}$ is a slowly hydrolyzed analog of ATP, ATP hydrolysis alone could not account for the differences between ATP and ATP $\gamma$ S in these RecA-NTD mutants. We also found that K23A (Figure 5) and three other NTD mutants (K23E, R33A, and R33E; data not shown) became catalytically active in D-loop formation upon mixing $\mathrm{AlF}_{4}^{-}$with ATP. $\mathrm{AlF}_{4}^{-}$was used here to trap the ADP-Pi transition state, because it is able to substitute for inorganic phosphate (Pi) after the hydrolysis of ATP. These results indicate that ATP $\gamma \mathrm{S}$ has a higher tendency than AMP-PNP to stabilize the activated transition state of RecA protein. ATP $\gamma S$ and AMP-PNP are known to have different structures among themselves, and in comparison with ATP or ADP-AlF - . Interestingly, GTP $\gamma \mathrm{S}$ and GMPPNP also exhibit similar diverse effects to some GTPases. For example, unlike GTP $\gamma$ S, GMP-PNP does not stabilize the activated transition state of Sar1 GTPase [22,23]. Sar1 is a structural component of coat complex II (COPII) vesicle coat, which coordinates the budding of transport vesicles from the endoplasmic reticulum in the initial step of the secretory pathway.

We then speculated that the wild-type, E18K, and E18K/ K23A proteins might be better than other NTD mutant proteins at converting ATP into ADP-Pi. Accordingly, we compared the ATPase activities of these three RecA proteins in response to ssDNA. The ssDNA-dependent ATPase activity was determined as described before, by quantification of the inorganic phosphate $(\mathrm{Pi})$ released from $\gamma^{32}$ P-labeled ATP [16]. Indeed, wild-type, E18K, and E18K/K23A proteins all exhibited higher ssDNA-dependent ATPase activity than the other four NTD mutants (K23A, K23E, R33A, and R33E) (Figure 4D). 

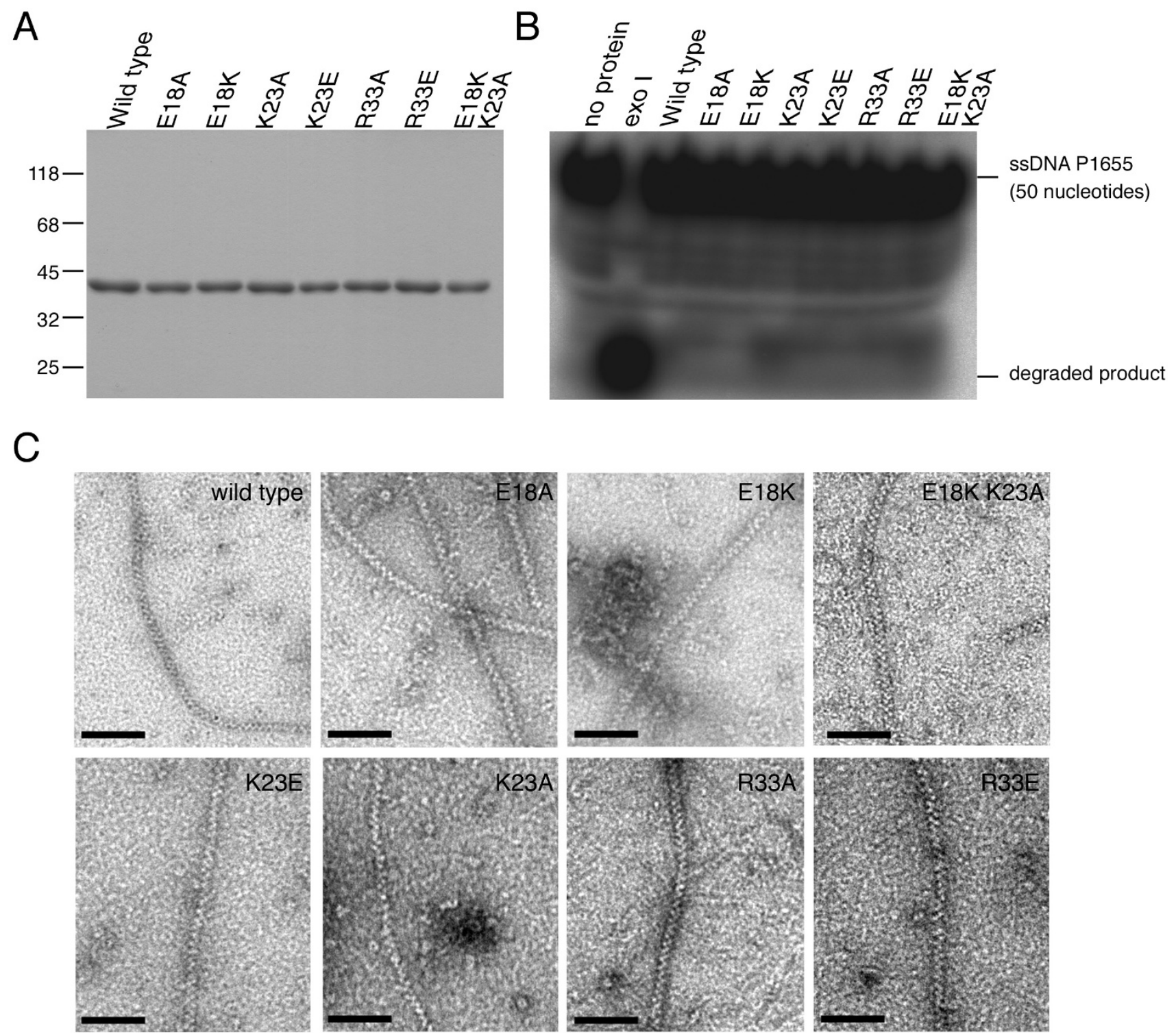

\section{Figure 3}

Production of wild-type and mutant RecA proteins. An improved SUMO fusion protein expression system was used to produce authentic RecA protein. (A) Purified RecA protein on a I \% SDS-PAGE gel stained with Coomassie Blue. (B) Nuclease activity assay. Purified RecA protein (I $\mu \mathrm{M})$ or exonuclease I (20 units; New England Biolabs) was incubated with 5'-end ${ }^{32} \mathrm{P}$ labeled PI656 ssDNA (50 nucleotides, $3 \mu \mathrm{M}$ ), respectively. The reaction mixtures were treated with proteinase $\mathrm{K}$ and then electrophoresed on $20 \%$ native acrylamide gels. Gels were visualized on a phosphorimager, with overexposure to confirm that the purified RecA proteins exhibited no detectable nuclease activity. (C) Purified RecA proteins are capable of polymerization into nucleoprotein helical filaments. Negative-staining electron microscopy images show various RecA proteins with a $\Phi \times 174$ dsDNA substrate in the presence of ATP $\gamma$ S. Scale bars (in black) are $100 \mathrm{~nm}$.

Taken together, the positively charged residues (Lys23, Arg33, or Lys 18 created by the E18K mutation) at the outer surface of the NTD could facilitate the RecA conversion of ATP into intermediate ADP-Pi in response to ssDNA. This conversion could be avoided with the use of
ATP $\gamma \mathrm{S}, \mathrm{ATP}_{-} \mathrm{AlF}_{4}{ }^{-}$, or even ADP-AlF${ }_{4}^{-}$. ATP $\gamma \mathrm{S}$ is known to be better than ATP or AMP-PNP at stabilizing the RecAssDNA presynaptic nucleoprotein filament $[1,12,24]$. However, Lys23 and Arg33 apparently make no direct contact with ssDNA or ATP in the crystal structure of the 

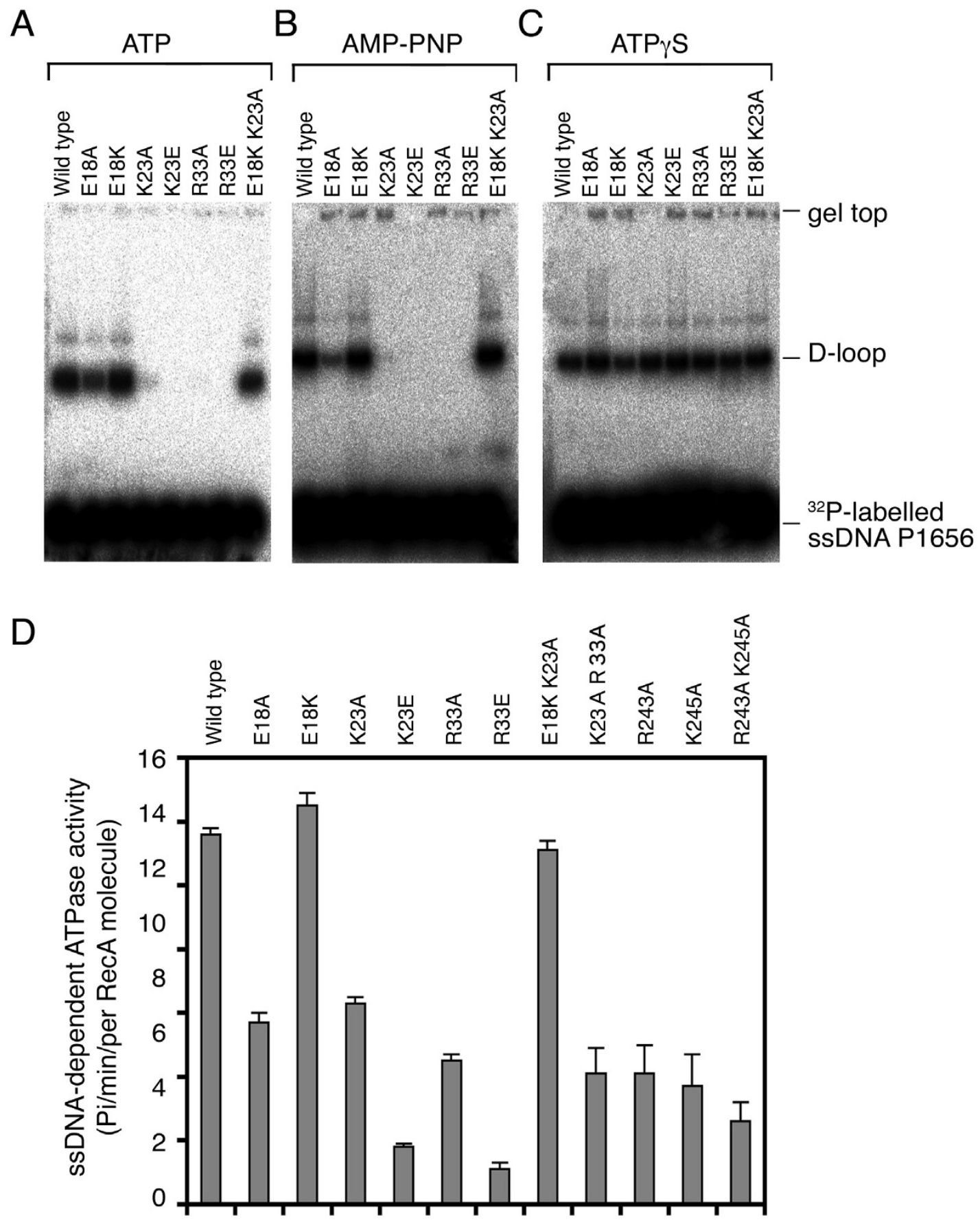

\section{Figure 4}

Functional characterization of wild-type and mutant RecA proteins. (A-C) D-loop formation. The ability to form a D-loop by RecA proteins was determined in the presence of ATP (A), AMP-PNP (B) or ATP $\gamma$ S (C). Reaction samples from the 10 min time point are shown. (D) The ATPase activities of RecA proteins in response to ssDNA. Wild-type or mutant RecA proteins $(0.5 \mathrm{mM})$ were incubated in the presence of $\mathrm{I} \mathrm{mM} \mathrm{Mg^{2+ }}$, with or without $\Phi$ XI74 ssDNA (I mM nucleotides). ATP hydrolysis was initiated by adding I mM ATP (with $0.6 \mathrm{nM}[\gamma-32 \mathrm{P}] \mathrm{ATP}$ ) at $37^{\circ} \mathrm{C}$. At different time points, $0.3 \mu \mathrm{L}$ aliquots were withdrawn and spotted on thin layer chromatography paper to separate [ $\gamma$ - $32 \mathrm{P}] \mathrm{ATP}$ from ${ }^{32} \mathrm{P}$-labeled inorganic phosphate. All RecA proteins examined here exhibited relatively low ATPase activities $(<0.5 \mathrm{Pi} / \mathrm{min} / \mathrm{RecA}$ protein) in the absence of ssDNA. Differences between RecA ATPase activities in the presence and absence of ssDNA are shown. 

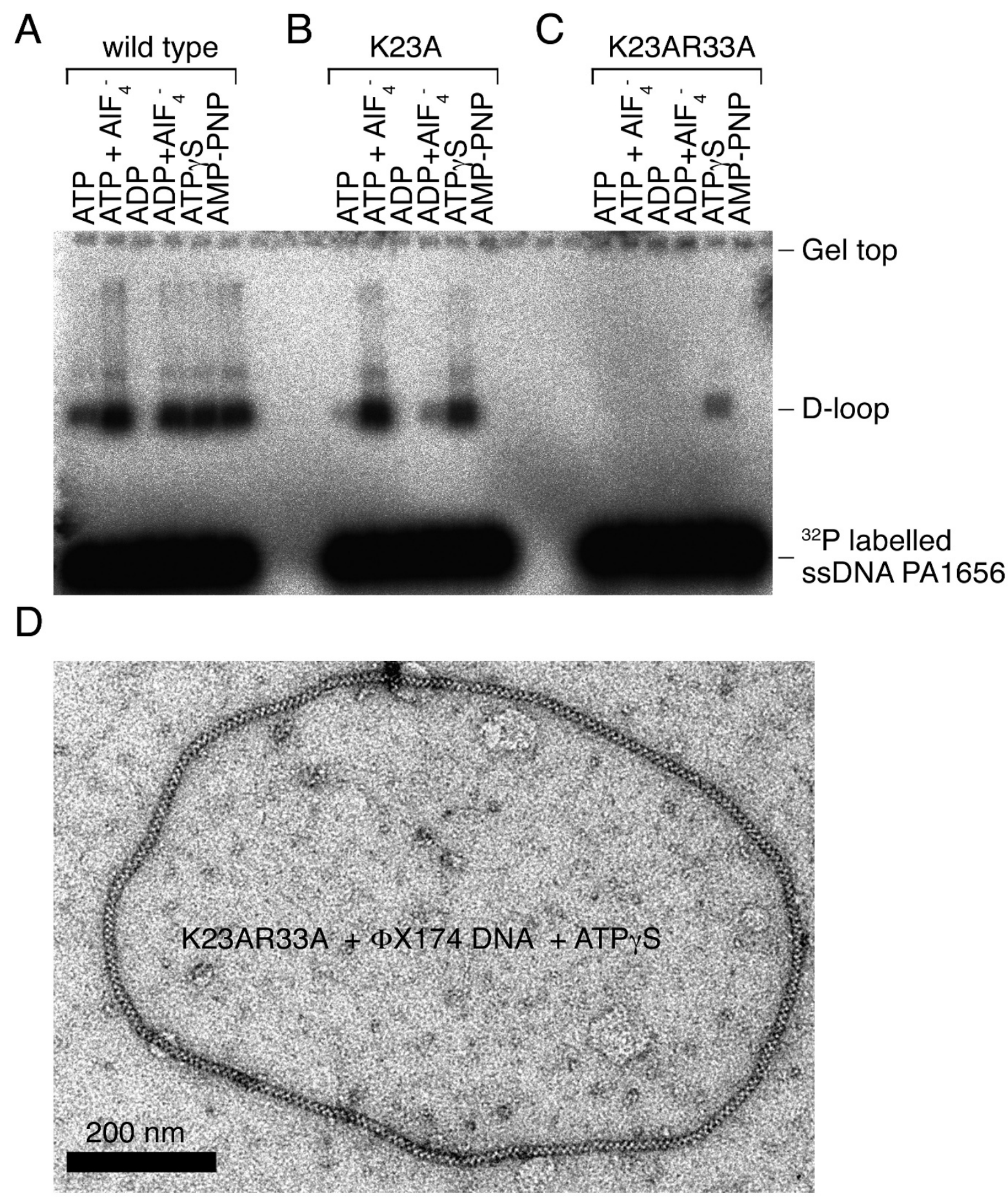

\section{Figure 5}

Effects of different nucleotide cofactors on wild-type (A), K23A (B), and K23A/R33A (C) RecA protein

mutants. D-loop formation in the presence of different nucleotide cofactors, as indicated, is shown. (D) K23A/R33A mutant proteins formed a presynaptic nucleoprotein filament with ssDNA in the presence of ATP $\gamma$. Negative-staining electron microscopy image shows K23A/R33A proteins with a circular $\Phi$ XI74 dsDNA in the presence of ATP $\gamma$ S. Scale bar (in black) is 200 $\mathrm{nm}$. 
RecA-ssDNA-ADP-AlF $4-\mathrm{Mg}^{2+}$ presynaptic nucleoprotein filament (Figure 1A) [12]. It is likely that these positively charged residues facilitate a RecA protein filament to search for and capture ssDNA in the presence of ATP, and to then convert ATP into intermediate ADP-Pi. As a result, the protein filament binds ssDNA tightly to form an active presynaptic nucleoprotein filament.

\section{Lys23 and Arg33 are indispensable for RecA's function in the presence of $A T P / S$}

We generated a K23A/R33A double mutant that, to our surprise, produced far less D-loop product in the presence of ATP $\gamma \mathrm{S}$ or $\mathrm{ATP}_{-} \mathrm{AlF}_{4}$ - (Figure 5). However, these three mutants exhibited similar ssDNA binding (data not shown) and ssDNA-dependent ATPase activities (Figure 4D). EM imaging analysis confirmed that this double mutant could still form a nucleoprotein filament with a circular ФX174 dsDNA, indicating that the K23A/R33A mutant was not defective in RecA polymerization or in formation of nucleoprotein filament (Figure 5D). Therefore, Lys23 and Arg33 together have an additional function in promoting D-loop formation, and this novel function may be similar to those of Arg243 and Lys245. As described above, Arg243 and Lys245 were implicated earlier as binding sites of donor dsDNA [12,14,15,25,26]. We then expressed and purified three additional mutants, R243A, K245A, and R243A/K245A. Like the single mutants, the K23A/R33A and R243A/K245A double mutants exhibited low ATPase activities in response to
ssDNA (Figure 4D). We found that the R243A/K245A double mutant, like K23A/R33A, produced very little or no D-loop product in the presence of ATP $\gamma \mathrm{S}$. By contrast, K243A and R245A single mutants, like K23A and R33A, still could produce D-loop products (Figure 6 ). The latter finding is consistent with a previous report that R243Q and $\mathrm{K} 245 \mathrm{~N}$ single mutants had only $33 \%$ and $66 \%$ of the activity, respectively, of the wild-type RecA [15].

\section{Lys23 and Arg33 are required for the RecA-ssDNA presynaptic nucleoprotein filament to capture dsDNA}

In the D-loop formation reaction, a RecA-ssDNA presynaptic filament must first engage the donor dsDNA molecule for the homology search and heteroduplex formation to occur. We thus performed a dsDNA capture assay according to a protocol (see Figure 7A for schematic) that was modified from one described previously [17]. The R243A/K245A double mutant was used as a negative control in this dsDNA capture assay. RecA, ATP $\gamma \mathrm{S}$, and bovine serum albumin (BSA) were first incubated with magnetic beads coated with 5'-biotinylated PA1656 ssDNA molecules to assemble stable presynaptic filaments. BSA was a negative control for ssDNA binding. To obtain equal amounts of RecA-ssDNA nucleoprotein filaments on the magnetic beads for the subsequent dsDNA capture assay, different amounts of wild-type protein $(5 \mu \mathrm{M})$ or each double mutant protein $(20 \mu \mathrm{M})$ were included. The ssDNA-RecA-ATP $\gamma \mathrm{S}$ complexes assembled on the magnetic beads were then isolated with a magnetic separator,

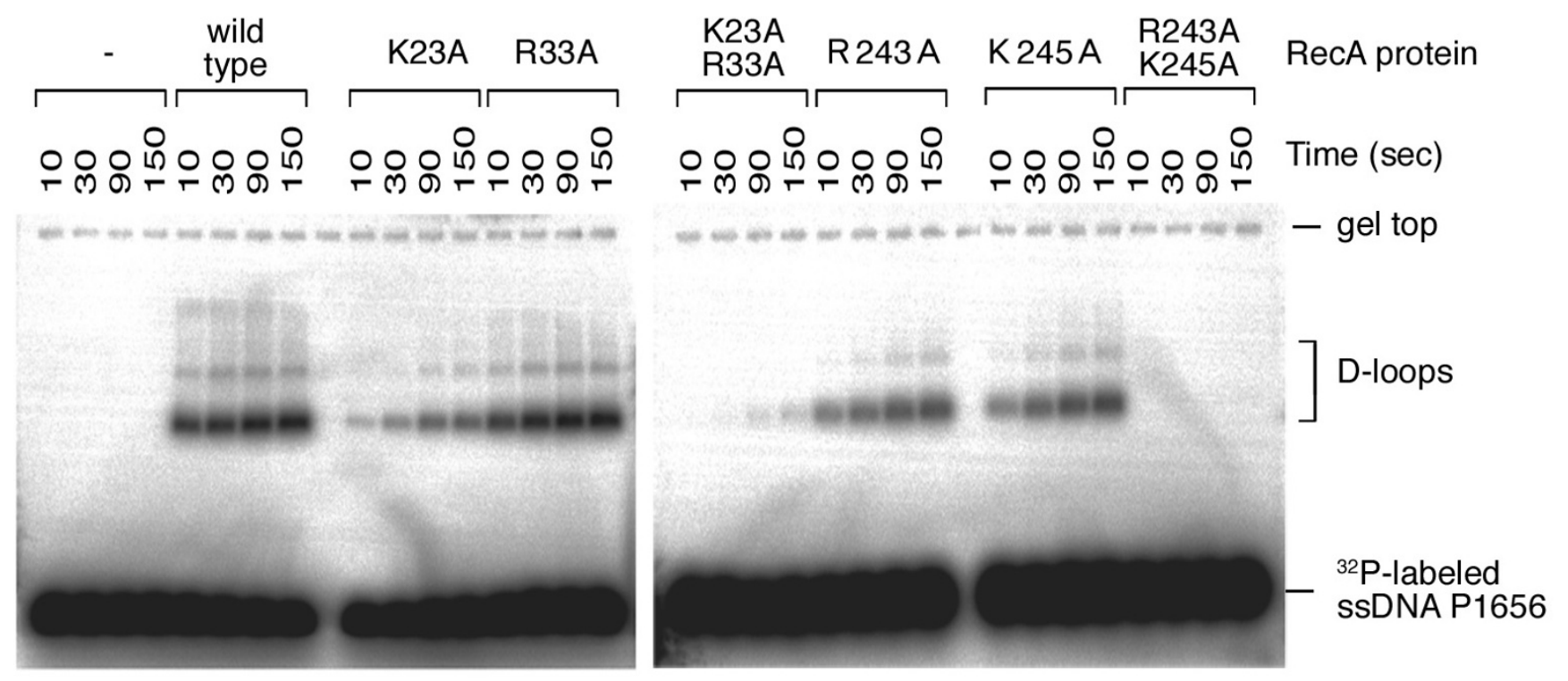

Figure 6

The K23A/R33A and R243A/K245A double mutants are catalytically inactive in promoting D-loop product formation. Time course analysis was performed in the presence of ATP $\gamma$. Aliquots were withdrawn from the reactions after I0, 30,90 , or 150 seconds, and then processed for analysis. 


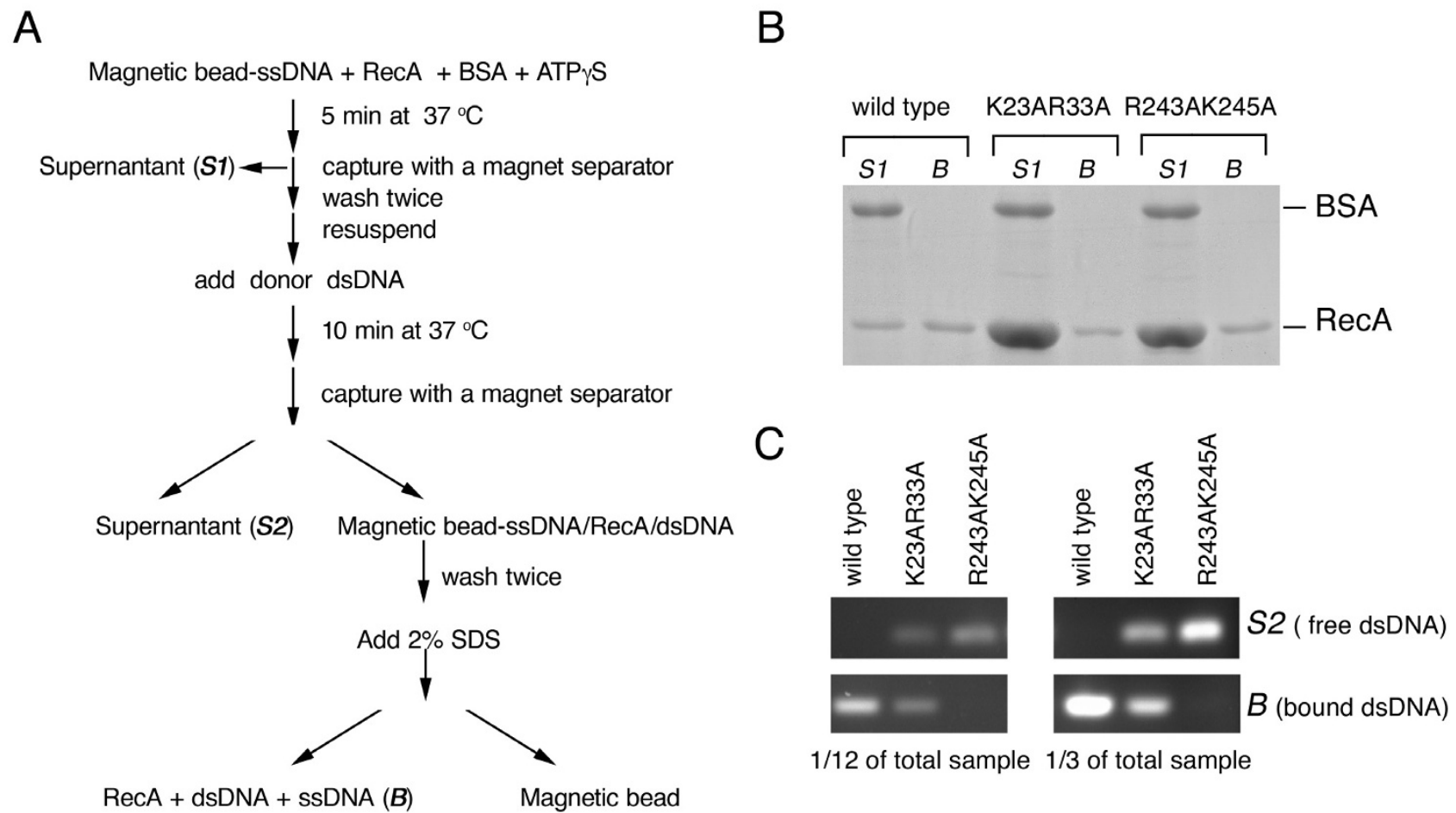

Figure 7

The presynaptic nucleoprotein filament of K23A/R33A mutant protein is defective in dsDNA capture. (A) Scheme of assay for examining DNA capture by presynaptic nucleotide protein filaments. See the main text and Materials and Methods for a detailed description. BSA was used as a negative control for ssDNA binding. R243A/K245A was a negative control for dsDNA capture by a presynaptic nucleoprotein filament. (B) SDS-PAGE analysis. RecA and BSA protein in "SI" and "B" were separated by electrophoresis in a $10 \%$ reducing polyacrylamide gel, and visualized by staining with Coomassie blue. (C) DNA agarose gel. For better quantitation, one-third or one-twelfth of the dsDNA (300 bps in length) in "S2" or "B" were separated on a $1 \%$ agarose gel, stained with ethidium bromide and then visualized by UV illumination.

with the supernatant (denoted as S1) retained for later analysis. The magnetic beads were then resuspended in a buffer containing ATP $\gamma$ S, BSA and a donor dsDNA for 10 min to initiate the homologous pairing or search reaction. This donor dsDNA was $300 \mathrm{bp}$ in length and contained a nucleotide sequence homologous to PA1656 ssDNA. Subsequently, a magnetic separator was used again to separate supernatant (denoted as "S2") and magnetic beadssDNA-RecA-dsDNA supercomplexes (denoted as "B"). B was then treated with $1 \%$ SDS to elute proteins and nucleic acids, which were then electrophoresed in a $1.5 \%$ non-denaturing agarose gel followed by staining with ethidium bromide and UV illumination to quantify the amount of dsDNA captured by the RecA-ssDNA presynaptic filaments. S1, S2 and B were also electrophoresed in a $10 \%$ denaturing polyacrylamide gel, followed by staining with Coomassie blue, to quantify RecA and BSA. Notably, S2 contained no or very little RecA protein, because the
RecA-ssDNA nucleoprotein filament was very stable in the presence of ATP $\gamma$ S (data not shown). BSA was only detected in the supernatant (S1) and did not bind to magnetic bead-ssDNA (Figure 7B). The DNA-agarose gel results indicate that the wild type RecA-ssDNA nucleoprotein filament was capable of capturing almost all donor dsDNA in the presence of ATPgS (Figure 7C). By contrast, $\mathrm{R} 243 \mathrm{~A} / \mathrm{K} 245 \mathrm{~A}$ and $\mathrm{K} 23 \mathrm{~A} / \mathrm{R} 33 \mathrm{~A}$ double mutant proteins captured $0 \%$ and $\sim 25 \%$ of total donor dsDNA, respectively.

Taken together, we conclude that both K23A/R33A and R243A/K245A mutants have two identical defects. First, both mutants had a lower binding affinity for ssDNA than the wild-type protein, because more mutant $(20 \mu \mathrm{M})$ than wild-type protein $(5 \mu \mathrm{M})$ was needed to assemble an equal amount of RecA-ssDNA presynaptic nucleoprotein filaments (Figure 7B). Second, once the RecA-ssDNA pre- 
synaptic filaments were assembled in the presence of ATP $\gamma$, both mutants were defective in capturing donor dsDNA (Figure 7C).

\section{Discussion}

We report here that the E. coli RecA NTD exhibits limited but significant amino acid sequence homology with the NTD (specifically, the second $\mathrm{H} 1 \mathrm{hH} 2$ motif) of the homologous proteins Rad51/Dmc1/RadA (Figure 2A). Notably, four basic residues (Lys8, Lys19, Lys23, and Arg33) of NTD constitute a positively charged helical patch along the outer surface of the RecA-ssDNA-ADP$\mathrm{AlF}_{4}-\mathrm{Mg}^{2+}$ nucleoprotein filament. We also found that these four basic residues at the NTD have at least two distinct roles in promoting RecA-mediated homologous recombination by capturing DNA. First, they help the RecA protein, in response to ssDNA, to convert ATP into intermediate $\mathrm{ADP}-\mathrm{Pi}$, a process that can be avoided by use of ATP $\gamma \mathrm{S}$ or ATP-AlF ${ }_{4}^{-}$. Then, the NTD facilitates the RecAssDNA presynaptic nucleoprotein filament capture of donor dsDNA during the homologous search reaction. We think this function may be similar to those of the NTDs in eukaryotic and archaeal RecA proteins [18,19].

The second function of the E. coli RecA NTD may be similar to that of Arg243 and Lys245. The NTD and Arg243/ Lys245 might function at the same time to facilitate an active RecA-ssDNA-ATP $\gamma$ S nucleoprotein filament to capture dsDNA. However, judging from the crystal structure of the RecA-ssDNA-ADP-AlF ${ }_{4}^{-}-\mathrm{Mg}^{2+}$ nucleoprotein filament (Figure 1) [12], it is unlikely for a donor dsDNA to simultaneously contact both Lys23/Arg33 and Arg243/ Lys245. This is because the basic amino acid residues of the NTD (including Lys8, Lys19, Lys23, and Arg33) are located along the outermost surface of the nucleoprotein filament, while Lys 243 and Arg245 are embedded in the center of the nucleoprotein filament (Figure 1B). Therefore, we propose the following model to explain their functions during D-loop formation reaction. After an active RecA-ssDNA nucleoprotein filament is assembled in the presence of ATP $\gamma \mathrm{S}$ (or ADP-Pi), the positively charged amino acid residues of the NTD will first non-specifically contact the phosphate groups of dsDNA, subsequently helping Lys243 and Arg245 to establish interactions with this donor dsDNA, and then carry out the strand exchange reaction. A prerequisite for this hypothesis is a rather large structural movement between the NTD and the CAD. This is not impossible. In fact, two similar incidents have been reported recently. First, during assembly of the RecA-ssDNA nucleoprotein filament, a hinge region that connects the NTD and CAD can undergo a large conformational change in response to ssDNA and $\mathrm{ADP}-\mathrm{AlF}_{4}-\mathrm{Mg}^{2+}$. This hinge region is located immediately after the polymerization motif, which functions as a fulcrum to mediate this large conformational change [12].
Second, we reported that clockwise axial rotation of the SRM of archaeal RadA proteins is responsible for a serial structural transition of RadA polymers from a protein ring to a right-handed helical filament, then to an over-wound right-handed helical filament, and finally to a left-handed helical filament. The SRM is located between the NTDs and CADs of RadA/Rad51/Dmc1 proteins, and it also uses the polymerization motif as a fulcrum to mediate this clockwise rotation along the helical filament axis $[10,11,18]$. It would be interesting to investigate whether the RecA NTD actually completes the rest of the dsDNA capture by rotating along the central axis of a presynaptic nucleoprotein filament.

Our results here are consistent with several previous studies on the RecA NTD. First, modeling of a 24-residue RecA $\mathrm{N}$-terminal peptide revealed that the four positively charged residues (Lys6, Lys8, Lys19, and Lys23) were capable of binding DNA phosphate groups by electrostatic interactions [27]. Second, it was reported that K6A/ K19A and K6A/K23A double mutants both exhibited severe defects in RecA function in vivo [28]. Third, deletion of the first N-terminal 9 amino acids of RecA (RecA- $\Delta 9$ ) manifested a rec phenotype [29]. The RecA- $\Delta 9$ protein loses both Lys 6 and Lys8, although the authors emphasized only the lack of Lys6. They then postulated that the $\Delta 9$ mutant was defective in mediating essential monomermonomer contact [29], because the $\varepsilon-\mathrm{NH}_{3}$ group of Lys 6 forms a salt bridge with the carboxylate oxygen of the Asp139 side chain in the neighboring subunit [3]. This hypothesis was correct for the free RecA protein filament, because the purified K6A mutant protein was indeed partly defective in oligomeric interaction in the absence of DNA [30]. The Lys6-Asp139 salt bridge also exists in the RecA-ssDNA-ADP-AlF ${ }_{4}^{-}-\mathrm{Mg}^{2+}$ nucleoprotein filament [12]. However, the K6A mutant protein demonstrated apparently normal formation of RecA-ssDNA helical filaments in vitro [30]. In addition, the recA-K6A and recAK6D mutants both exhibited a rec ${ }^{+}$phenotype [30]. Therefore, a decrease in monomer-monomer contact due to breakage of the Lys6-Asp139 salt bridge is not likely to be the primary cause for the rec phenotype of $\Delta 9, K 6 \mathrm{~A} / \mathrm{K} 19 \mathrm{~A}$, and K6A/K23A mutants. Since the basic side chains of Lys8, Lys19, and Lys23 are located at the outer surface of RecA or RecA-ssDNA filaments to bind ssDNA or dsDNA, we think the rec phenotype of $\Delta 9, \mathrm{~K} 6 \mathrm{~A} / \mathrm{K} 19 \mathrm{~A}$, and K6A/ K23A mutants may be the result of a loss or mutation of Lys8, Lys19, or Lys23, respectively.

In conclusion, we showed in this report that the NTD of RecA protein sequentially mediates ssDNA and dsDNA binding during homologous recombination. Our results also imply that a rather large rigid body movement between the NTD and CAD may be required for an active RecA-ssDNA-ATP $\gamma \mathrm{S}-\mathrm{Mg}^{2+}$ nucleoprotein filament to carry 
out homology searching and binding to a target dsDNA. We suggest that such a rigid body movement is mediated by axial rotation of the NTD and CAD along the central axis of the RecA protein filament.

\section{Competing interests}

The authors declare that they have no competing interests.

\section{Authors' contributions}

CDL carried out all experiments and analyzed the data. TFW conceived and designed the experiment, analyzed the data, wrote the paper and the principle investigator. Both authors read and approved the final manuscript.

\section{Acknowledgements}

This work was supported by Academia Sinica (AS-97-FP-M02 to TFW) and the National Science Council, Taiwan (NSC96-232I-B-00I-019 to TFW). We thank Dr. Yuan-Chih Chang (Institute of physics, Academia Sinica) for help in EM imaging.

\section{References}

I. Cox MM: The bacterial RecA protein as a motor protein. Annu Rev Microbiol 2003, 57:55I-577.

2. Hastings PJ, Rosenberg SM, Slack A: Antibiotic-induced lateral transfer of antibiotic resistance. Trends Microbiol 2004, 1 2:40|-404.

3. Story RM, Weber IT, Steitz TA: The structure of the E. coli RecA protein monomer and polymer. Nature 1992, 355:3।8-325.

4. Wang JM: Nucleotide-dependent domain motions within rings of the RecA/AAA+ superfamily. J Struct Biol 2004, 148:259-267.

5. Story RM, Steitz TA: Structure of the RecA protein-ADP complex. Nature 1992, 355:374-376.

6. Chen PL, Chen CF, Chen Y, Xiao J, Sharp ZD, Lee WH: The BRC repeats in BRCA2 are critical for RAD5 I binding and resistance to methyl methanesulfonate treatment. Proc Nat Acad Sci 1998, 95:5287-5292.

7. Yuan SS, Lee SY, Chen G, Song M, Tomlinson GE, Lee EY: BRCA2 is required for ionizing radiation-induced assembly of Rad5 I complex in vivo. Cancer Res 1999, 59:3547-355I.

8. Pellegrini L, Yu DS, Lo T, Anand S, Lee M, Blundell TL, Venkitaraman $A R$ : Insights into DNA recombination from the structure of a RAD5I-BRCA2 complex. Nature 2002, 420:287-293.

9. Shin DS, Pellegrini L, Daniels DS, Yelent B, Craig L, Bates D, Yu DS, Shivji MK, Hitomi C, Arvai AS, Volkmann N, Tsuruta H, Blundell TL, Venkitaraman AR, Tainer JA: Full-length archaeal Rad5 I structure and mutants: mechanisms for RAD5I assembly and control by BRCA2. EMBO J 2003, 22:4566-4576.

10. Chen LT, Ko TP, Chang YC, Lin KA, Chang CS, Wang AH, Wang TF: Crystal structure of the left-handed archaeal RadA helical filament: identification of a functional motif for controlling quaternary structures and enzymatic functions of RecA family proteins. Nucleic acids Res 2007, 35: I787-I80I.

II. Wang TF, Chen LT, Wang AH: Right or left turn? RecA family protein filaments promote homologous recombination through clockwise axial rotation. BioEssays 2008, 30:48-56.

12. Chen Z, Yang H, Pavletich NP: Mechanism of homologous recombination from the RecA-ssDNA/dsDNA structures. Nature 2008, 453:489-484.

13. Kowalczykowski SC: Structural biology: snapshots of DNA repair. Nature 2008, 453:463-466.

14. Rehrauer WM, Kowalczykowski SC: The DNA binding site(s) of the Escherichia coli RecA protein. J Biol Chem 1996, 27I:I I 996-I2002.

15. Kurumizaka H, Ikawa S, Sarai A, Shibata T: The mutant RecA proteins, RecAR243Q and RecAK245N, exhibit defective DNA binding in homologous pairing. Arch Biochem Biophys 1999, 365:83-91.

16. Lee CD, Sun HC, Hu SM, Chiu CF, Homhuan A, Liang SM, Leng CH, Wang TF: An improved SUMO fusion protein system for effective production of native proteins. Protein Sci 2008, 17:124I-1248.

17. Chi P, San Filippo J, Sehorn MG, Petukhova GV, Sung P: Bipartite stimulatory action of the Hop2-Mnd I complex on the Rad5 I recombinase. Genes Develop 2007, 21:1747-1757.

18. Chen LT, Ko TP, Chang YW, Lin KA, Wang AH, Wang TF: Structural and functional analyses of five conserved positively charged residues in the $\mathrm{LI}$ and $\mathrm{N}$-terminal DNA binding motifs of archaeal RADA protein. PLoS One 2007, 2:e858.

19. Aihara H, Ito Y, Kurumizaka H, Yokoyama S, Shibata T: The N-terminal domain of the human Rad5 I protein binds DNA: structure and a DNA binding surface as revealed by NMR. J Mol Biol 1999, 290:495-504

20. Karlin S, Brocchieri L: Evolutionary conservation of RecA genes in relation to protein structure and function. J Bacteriol 1996, 178:188I-I894

21. McGrew DA, Knight KL: Molecular design and functional organization of the RecA protein. Crit Rev Biochem Mol Biol 2003, 38:385-432.

22. Bi X, Corpina RA, Goldberg J: Structure of the Sec23/24-Sar I pre-budding complex of the COPII vesicle coat. Nature 2002, 419:27I-277.

23. Bielli A, Haney CJ, Gabreski G, Watkins SC, Bannykh SI, Aridor M: Regulation of SarI $\mathrm{NH}_{2}$ terminus by GTP binding and hydrolysis promotes membrane deformation to control COPII vesicle fission. J Cell Biol 2005, I 7 I:919-924.

24. Joo C, McKinney SA, Nakamura M, Rasnik I, Myong S, Ha T: Realtime observation of RecA filament dynamics with single monomer resolution. Cell 2006, I 26:5।5-527.

25. Kurumizaka H, Aihara H, lkawa S, Kashima T, Bazemore LR, Kawasaki $\mathrm{K}$, Sarai A, Radding CM, Shibata T: A possible role of the C-terminal domain of the RecA protein. A gateway model for double-stranded DNA binding. J Biol Chem 1996, 27 I:335 I5-33524.

26. Aihara $\mathrm{H}$, Ito $\mathrm{Y}$, Kurumizaka $\mathrm{H}$, Terada $\mathrm{T}$, Yokoyama $\mathrm{S}$, Shibata T: An interaction between a specified surface of the C-terminal domain of RecA protein and double-stranded DNA for homologous pairing. J Mol Biol 1997, 274:213-22I.

27. Zlotnick A, Brenner SL: An alpha-helical peptide model for electrostatic interactions of proteins with DNA. The $\mathbf{N}$ terminus of RecA. J Mol Biol 1988, 209:447-457.

28. Morimatsu K, Horii T: Analysis of the DNA binding site of Escherichia coli RecA protein. Adv Biophys 1995, 31:23-48.

29. Zaitsev EN, Kowalczykowski SC: Essential monomer-monomer contacts define the minimal length for the $\mathbf{N}$-terminus of RecA protein. Mol Microbiol 1998, 29:1317-1318.

30. Eldin S, Forget AL, Lindenmuth DM, Logan KM, Knight KL: Mutations in the $\mathrm{N}$-terminal region of RecA that disrupt the stability of free protein oligomers but not RecA-DNA complexes. J Mol Biol 2000, 299:9I-101.

\section{Publish with Biomed Central and every scientist can read your work free of charge}

"BioMed Central will be the most significant development for disseminating the results of biomedical research in our lifetime. "

Sir Paul Nurse, Cancer Research UK

Your research papers will be:

- available free of charge to the entire biomedical community

- peer reviewed and published immediately upon acceptance

- cited in PubMed and archived on PubMed Central

- yours - you keep the copyright

Submit your manuscript here:

http://www.biomedcentral.com/info/publishing_adv.asp
BioMedcentral 